\title{
Fatigue notch sensitivity as a function of the notch to grain size relationship
}

\author{
Pablo Lorenzino ${ }^{1, \mathrm{a}}$ and Alfredo Navarro ${ }^{2}$ \\ ${ }^{1}$ Mateis, INSA de Lyon, 20, Avenue Albert Einstein, 69621 Villeurbanne Cedex, France \\ ${ }^{2}$ Department of Mechanical Engineering, University of Seville, 41092 Seville, Spain
}

\begin{abstract}
This work presents experimental results showing the mode I fatigue behaviour of holed components as a function of the hole to grain size relationship. A wide range of notch size to grain size ratios are used, including cases where the notch size is on the order of, or even smaller, than the grain size. Making through-holes in the order of the grain size is very difficult in practice. So the technique used in this paper is to manufacture materials with very large grain size, on the order of a millimetre or even centimetre, then usual machines and tools can be used for making the notches. After the specimens are obtained and tested, the notch sensitivity is evaluated and it is shown how the notch sensitivity index of any material must always be defined in terms of its microstructure. It was found that for the same specimen geometry and loading conditions and identical stress concentrators, the influence of the stress concentrator is directly dependent on the relationship between their dimension and the grain size.
\end{abstract}

\section{Material and Test set-up}

Previous work has demonstrated that it is feasible to grow until the centimetre range the grain size of commercially pure aluminium sheets by a combination of two heat treatments and an intermediate cold deformation. The technique has been shown to produce highly consistent and repeatable results [1, 2]. In order to study the notch sensitivity as a function of grain size, specimens of five different grain sizes were used $(0.066-0.39-1.41-3.46-9.74 \mathrm{~mm})$. For each grain size, four sets of specimens with different hole sizes were obtained, namely, unnotched, 1, 2 and 4 or $6 \mathrm{~mm}$ diameter through-thickness circular notches. Thus $20 \mathrm{~S}-\mathrm{N}$ diagrams were obtained for the 20 different Notch-Size to Grain- ratios, varying from $D_{\text {Notch }} / D_{\text {Grain }}=0.1$ to $D_{\text {Notch }} / D_{\text {Grain }}=100$.

\section{Notch sensitivity analisys}

Figure 1 shows the obtained fatigue limits as a function of the notch to grain size relationship $D_{\text {Notch }} / D_{\text {Grain }}$. These fatigue limits are reported as the value of $\sigma \max$ under which the specimens do not fail after $1 \times 10^{6}$ cycles or as the normalized value of the fatigue limit, according to the fatigue limit of the unnotched component, $\sigma_{N o t c h ~}^{f l} / \sigma_{\text {Unnotch } f l}$, which is precisely $1 / K f$. In the graphs is clearly observed

\footnotetext{
${ }^{a}$ Corresponding author: pablo.lorenzino@insa-lyon.fr
}

This is an Open Access article distributed under the terms of the Creative Commons Attribution License 4.0, which permits unrestricted use, distribution, and reproduction in any medium, provided the original work is properly cited. 

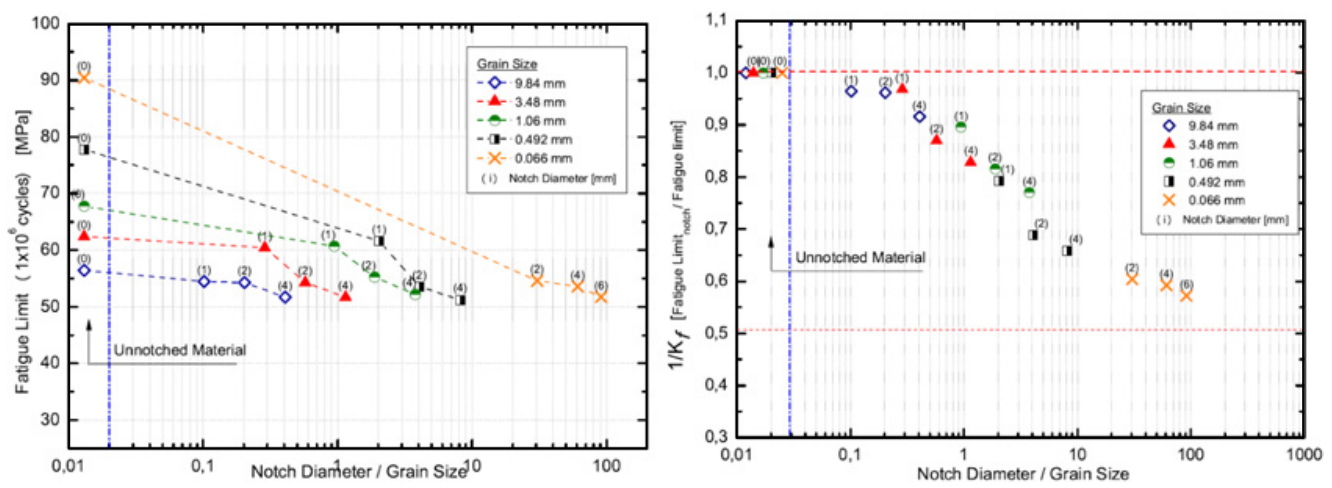

Figure 1. Fatigue limits and normalized fatigue limit for the different grain size as a function of the $D_{\text {Notch }} / D_{\text {Grain }}$ relationship.
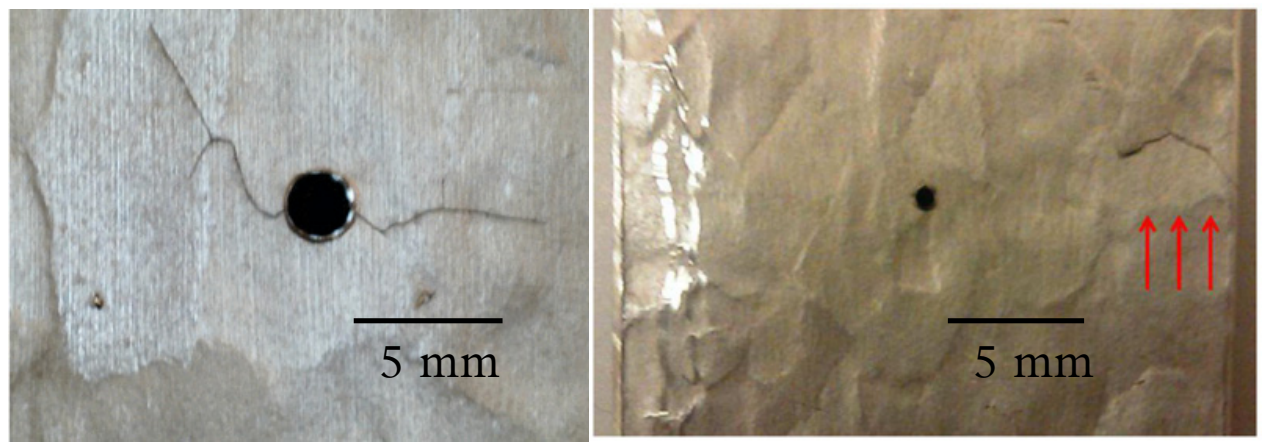

Figure 2. Fatigue cracks in different specimens.

that the decrease of fatigue limit depends not only on the geometry of the notch, but the relationship between their dimension and the grain size. For the same notch size and specimen geometry, the notch sensitivity depends upon the grain size. Of course, this was expected from the classical dependence of the notch sensitivity index upon the ultimate strength of the material familiar to every fatigue designer.

Furthermore, Fig. 2 shows on the left a crack growing from a $2 \mathrm{~mm}$ diameter circular notch in a $3.46 \mathrm{~mm}$ grain size microstructure, the cracks are not symmetrical, presenting a configuration characteristic of a microstructurally short fatigue crack. The figure on the left corresponds to a $1 \mathrm{~mm}$ circular notch in a $3.46 \mathrm{~mm}$ grain size microstructure, cycles to failure, $1.69 \times 10^{7}$. The crack, although it is located in the reduction of area due to the presence of the notch, does not originate in the stress concentrator. Probably the origin takes place in a better oriented grain (slip system) according to the loading axis of the material.

\section{Conclusions}

The actual physical size of the notch is not as important as the relative size of the notch with respect to the characteristic microstructural dimension.

\section{References}

[1] P. Lorenzino, A. Navarro, U. Krupp, Int. J. Fatigue. 56 (2013)

[2] P. Lorenzino, PhD Thesis, Seville University (2012) 